\title{
Expanding spectrum of "spitzoid" lesions: a small series of 4 cases with MAP2K1 mutations
}

\author{
K. G. P. Kerckhoffs ${ }^{1}$ (I) - T. Aallali ${ }^{2}$ - C. A. Ambarus ${ }^{3}$ - V. Sigurdsson ${ }^{4}$ - A. M. L. Jansen ${ }^{5}$ - W. A. M. Blokx ${ }^{5}$
}

Received: 12 June 2020 / Revised: 10 September 2020 / Accepted: 27 September 2020 / Published online: 11 October 2020

(C) The Author(s) 2020

\begin{abstract}
The molecular background of a significant proportion of spitzoid neoplasms is still unknown. Recently, activating mutations in MAP2K1 have been described in a few spitzoid lesions, but not in benign Spitz nevi. We report four cases of melanocytic tumors with spitzoid features in which a MAP2K1 mutation was detected. The lesions did not show a single distinct phenotype and ranged from benign to malignant. Two cases resembled desmoplastic Spitz nevi. Based on the combination of morphological, immunohistochemical, and molecular findings, one case was classified as benign, one as probably benign, possibly intermediate low-grade (MELTUMP - melanocytic tumor of unknown malignant potential), one case was classified as intermediate (MELTUMP), and one case was considered a superficial spreading melanoma with spitzoid features. Based on this, we conclude that MAP $2 K 1$ mutations can indicate a spitzoid genetic signature and can be found in both benign and malignant spitzoid neoplasms.
\end{abstract}

Keywords Spitz nevus $\cdot$ MELTUMP $\cdot$ Melanoma $\cdot$ MAP2K1

\section{Background}

During recent years, great progress has been made in analyzing the molecular background of melanocytic lesions, including spitzoid neoplasms. Spitzoid neoplasms are defined based on their histopathologic appearance. They show large epithe-

Electronic supplementary material The online version of this article (https://doi.org/10.1007/s00428-020-02940-3) contains supplementary material, which is available to authorized users.

K. G. P. Kerckhoffs

kelly.kerckhoffs@mumc.nl

1 Department of Pathology, Maastricht University Medical Center+, Maastricht, The Netherlands

2 Symbiant Pathology Expert Center, Hoorn/Zaandam, The Netherlands

3 Department of Pathology, Sint Antonius Hospital, Nieuwegein, The Netherlands

4 Department of Dermatology, University Medical Center Utrecht, Utrecht, The Netherlands

5 Department of Pathology, Division of Laboratories, Pharmacy and Biomedical Genetics, University Medical Center Utrecht, Utrecht, The Netherlands lioid or spindled cells with specific architectural features. It was known that about $20 \%$ of the spitzoid tumors carry an HRAS mutation [1]. More recently, chromosomal rearrangement-induced fusions have been identified in 50 to $55 \%$ of spitzoid lesions involving the kinase genes ROS1, ALK, BRAF, NTRK1, NTRK3, MET, and RET [2, 3]. While the genetics of a significant portion of spitzoid neoplasms has been identified, in a significant portion of cases, the driver event is still unknown. Quan et al. recently identified structural rearrangements in MAPK genes other than $B R A F$ in eight spitzoid neoplasms [4]. Among these, they identified one case diagnosed as an atypical Spitz tumor (AST) with an activating in-frame deletion in MAP2K1. MAP2K1 encodes MEK1, a serine-threonine and tyrosine kinase directly downstream of RAF. This kinase phosphorylates and activates ERK1/2 [5, 6]. Activating mutations in $M A P 2 K 1$ have been identified in melanoma [7, 8]. Raghavan et al. reported a MAP2K1 mutation in a case they classified as spitzoid melanoma (malignant Spitz tumor, MST) [9]. Besides this, mutations in MAP2K1 have been described in deep penetrating nevi (DPN) in combination with mutations in the beta-catenin pathway [10].

Herein, we report four cases of melanocytic tumors with spitzoid features in which a pathogenic MAP2K1 mutation was detected. 


\section{Material and methods}

The four cases were identified during routine molecular workup of spitzoid lesions. Three cases were sent in consultation to the Pathology Department of the University Medical Center in Utrecht (UMCU) (consultant pathologist WB). One case was initially seen in the Symbiant Pathology Expert Center in Alkmaar, one in the Meander Medical Center in Amersfoort, and one in the Sint Antonius Hospital in Nieuwegein. The fourth case was initially seen in the UMCU. Details about the immunohistochemical stains that were used can be found in Online Resource 1.

DNA was isolated from formalin-fixed paraffin-embedded (FFPE) specimens using the cobas ${ }^{\circledR}$ DNA Sample Preparation Kit (Roche) (cases 1-3) or the Maxwell RSC 48 instrument (Promega) (case 4) using the Maxwell® RSC FFPE Plus DNA Purification Kit (Promega), according to the manufacturer's protocol. Subsequently, next-generation sequencing (NGS) and single nucleotide polymorphism (SNP) array analysis were performed (for details, see Online Resource 2).

\section{Case presentation}

Detailed clinical information, microscopical and immunohistochemical findings, and the results of molecular testing of all four cases with a MAP2K1 mutation can be found in Tables 1 and 2. None of the cases showed additional mutations. Furthermore, relevant images of cases 2 and 3 can be found in Figs. 1 and 2 (images of cases 1 and 4 are available in Online Resource 3). For cases 3 and 4, clinical pictures were available, see Online Resource 4.

\section{Case 1}

Initially, based on morphology, a dermal desmoplastic Spitz nevus was considered, but no HRAS mutation was detected. The lesion did not show cytomorphologic features of a DPN. Since multiple copy number variations (CNVs) were detected with SNP array (3 CNVs and chromothripsis of chromosome $22 \mathrm{q}$ ), the lesion could not be classified as fully benign since presence of 3 or more $\mathrm{CNVs}$ is considered as indicative for malignancy [11]. However, morphology and immunohistochemistry did not clearly indicate malignancy. Therefore, the lesion was classified as an intermediate melanocytic tumor (MELTUMP - melanocytic tumor of unknown malignant potential-perhaps an AST). At the time of diagnosis of this lesion, no $M A P 2 K 1$ mutations in spitzoid lesions were reported in literature. The margins were free. A re-excision was performed showing no residual tumor.

\section{Case 2}

We considered this proliferation having spitzoid morphology, again with a $M A P 2 K 1$ mutation. In this case, there was loss of p16 staining. No features of a DPN were seen. Based on the six CNVs detected, it was decided to consider and treat the lesion as superficial spreading melanoma with spitzoid features (pTNM stage pT1a, TNM 8th Ed. 2017), and a reexcision with a margin of $1 \mathrm{~cm}$ was performed. In the reexcision specimen, no residual tumor was detected. No sentinel node procedure was conducted.

\section{Case 3}

This clinical atypical nevus (for clinical pictures, see Online Resource 4) showed spitzoid morphology with features of a Reed nevus. The single mitotic figure and focal ascent of melanocytes within the epidermis were considered a consequence of the encountered inflammation. With maintained expression of p16 and lack of CNVs, we had no suspicion of an intermediate or malignant lesion. Therefore, the lesion was classified as a variant Spitz nevus with features of pigmented spindle cell nevus of Reed with inflammatory changes and mild reactive atypia. Since the margins were free, no additional treatment was necessary.

\section{Case 4}

Clinically, the lesion was present for at least 4 years and unchanged (also based on repeated dermatological examination and unchanged clinical pictures, see Online Resource 4) without suspicion for malignancy. Also, based on cytomorphology, this melanocytic proliferation was regarded benign. A spindle cell nevus or desmoplastic Spitz nevus was considered. The lesion did not show classic DPN morphology. SNP array showed trisomy of chromosomes 5, 6, 11, 14, 15, and 20. Due to poor quality of the SNP array, presence of other CNVs could not be excluded completely. Gains of entire chromosomes in the absence of structural aberrations have been described as compatible with benign lesions in congenital nevi [12], outside the context of congenital nevi the meaning of numerical aberrations is unclear. Therefore, the lesion was classified as probably benign, at most low-grade MELTUMP (perhaps AST). A re-excision was performed showing no residual tumor.

\section{Discussion}

We report four MAP2K1-mutated melanocytic tumors with different morphology ranging from benign to malignant but all sharing spitzoid morphological features. In about $20 \%$ of melanocytic neoplasms, including a proportion of spitzoid 


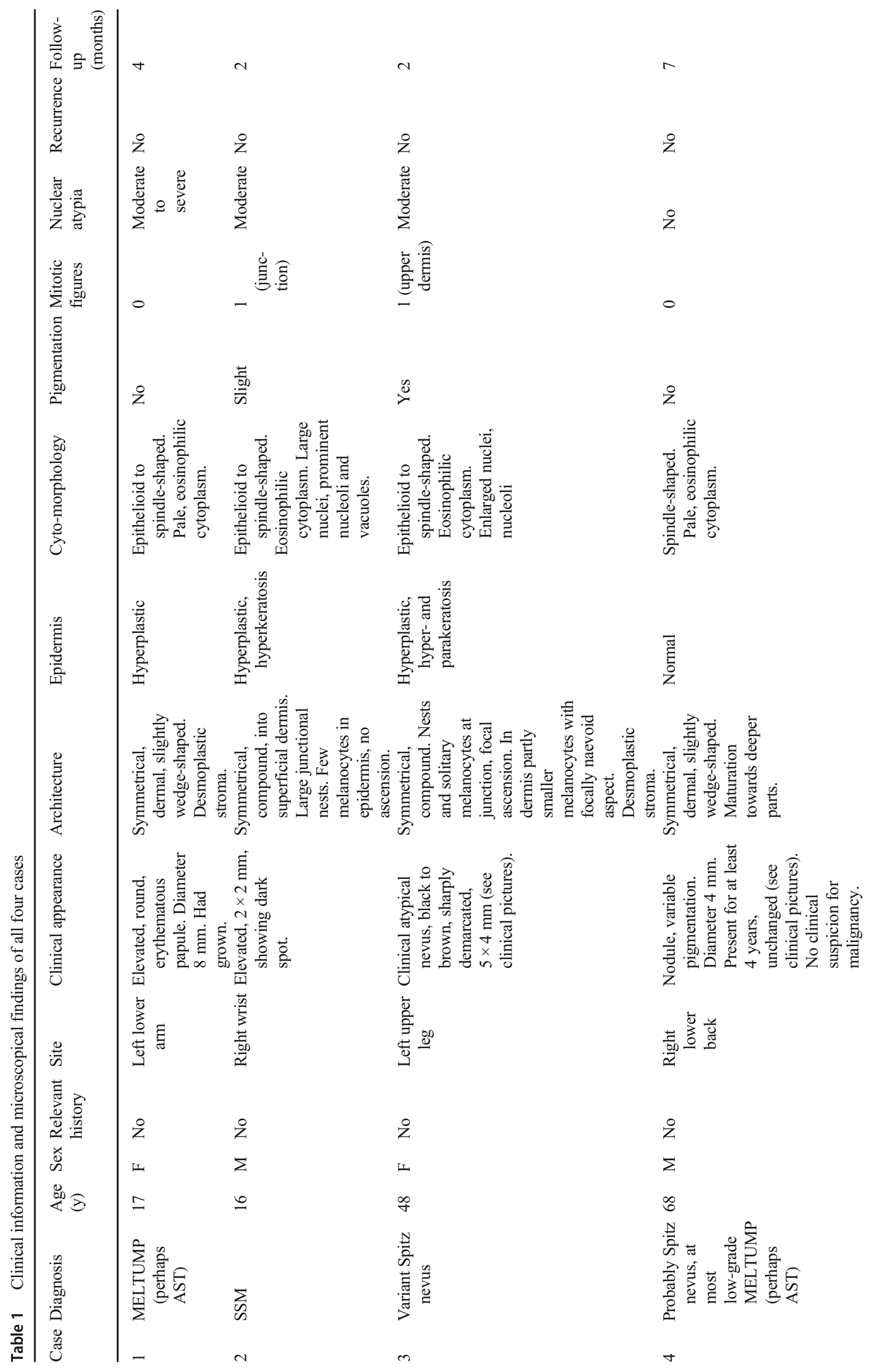

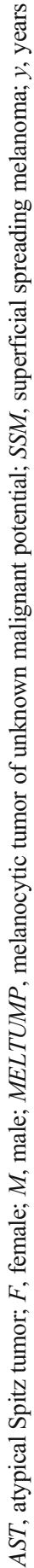




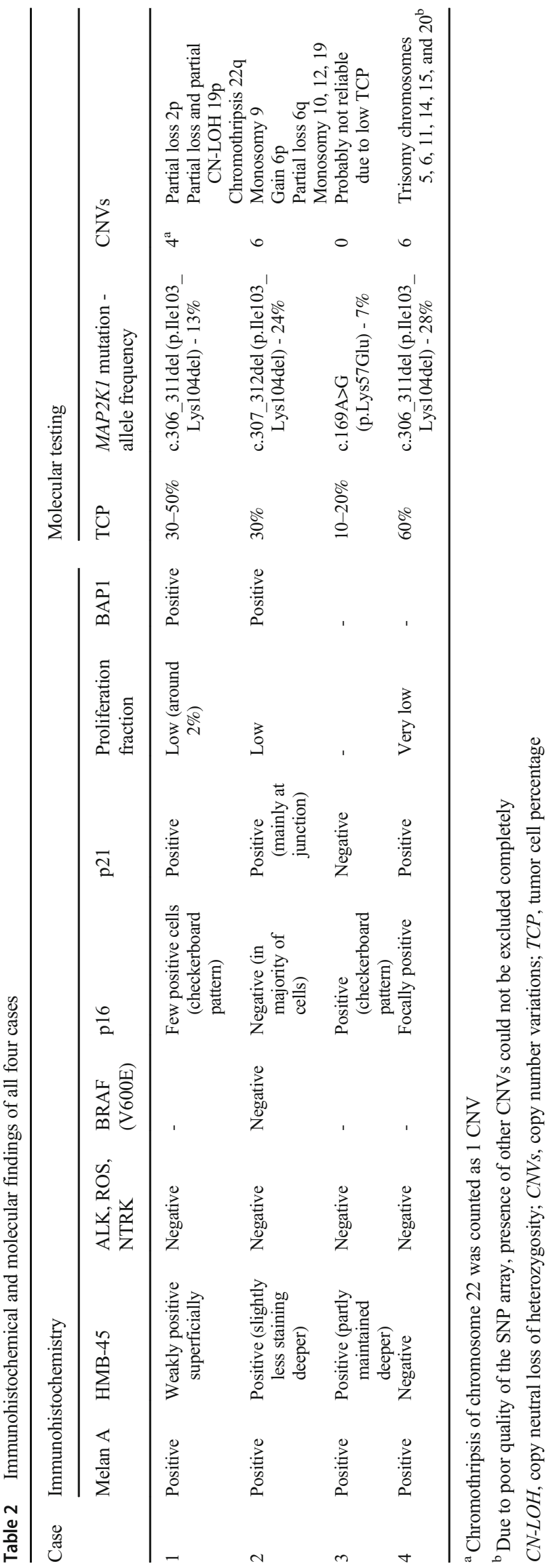

lesions, the genomic driver is thus far unknown. Despite the use of a broad panel of additional tests including NGS, immunohistochemistry, fluorescence in situ hybridization (FISH), or Archer techniques, no mutations, translocations, or amplifications can be demonstrated in a part of the spitzoid neoplasms. MAP2K1 was added to the NGS panel in our practice on September 24, 2019. Since this date, 174 NGS analyses were performed on melanocytic lesions. The four reported cases were the first spitzoid neoplasms identified in our practice showing a pathogenic $M A P 2 K 1$ mutation, indicating a $M A P 2 K 1$ mutation is a rare genomic driver in these neoplasms. We consider the morphology of these lesions as clearly spitzoid and, in the absence of other specific mutations or translocations, we consider it highly likely that the MAP2K1 mutation is the underlying genomic driver. We could not discern a clearly distinct phenotype within these MAP2K1-mutated lesions, although two cases resembled desmoplastic Spitz nevi. Furthermore, our four cases did not show a distinct phenotype compared with $M A P 2 K 1$ wild-type spitzoid lesions.

In the 4th edition of the 2018 WHO classification of skin tumors [13], lesions in the spitzoid pathway (pathway 4) are characterized by mutations in $H R A S$, tyrosine kinase fusions (ALK, ROS1, RET, NTRK1/3, and MET), or serine-threonine kinase fusions (BRAF, MAP3K8). It also mentions the presence of MAP2K1 mutations in DPN lesions (pathway 1).

Up to now, $M A P 2 K 1$ alterations have hardly been reported in spitzoid melanocytic lesions, and $M A P 2 K 1$ alterations have not been reported in benign Spitz nevi yet. Last year, two spitzoid lesions with $M A P 2 K 1$ alterations were reported; one case of an AST [4] and one case of MST [9]. Our small case series confirms $M A P 2 K 1$ mutations can be present in lesions with a spitzoid morphology, and also shows the presence of this mutation in two (probably) benign cases.

Histologically, we saw relatively symmetrical melanocytic proliferations consisting of epithelioid to spindle-shaped cells. Cases 1, 2, and 4 demonstrated a comparable MAP2K1 mutation resulting in the same deletion on protein level (p.Ile103_Lys104del). In case 1, desmoplastic stroma was seen and molecular diagnostics revealed $3 \mathrm{CNVs}$ and chromothripsis of chromosome 22q, leading to classification as an intermediate lesion. Case 2 showed more cytonuclear atypia with immunohistochemical loss of p16; 6 CNVs were detected. Therefore, this lesion was classified as melanoma and treated as such. Case 4 did not show cytonuclear atypia or immunohistochemical signs of malignancy. SNP array revealed trisomy of 6 chromosomes without structural aberrations, based on which the lesion was classified as probably benign, intermediate at most. The same deletion (p.Ile103 Lys104del) was described by Quan et al. in an AST [4]. Amino acids 98 to 104 of MEK1 comprise an autoinhibitory domain, so a mutation in this region leads to activation of ERK that does not respond to feedback inhibition by RAS and RAF [4]. 
a

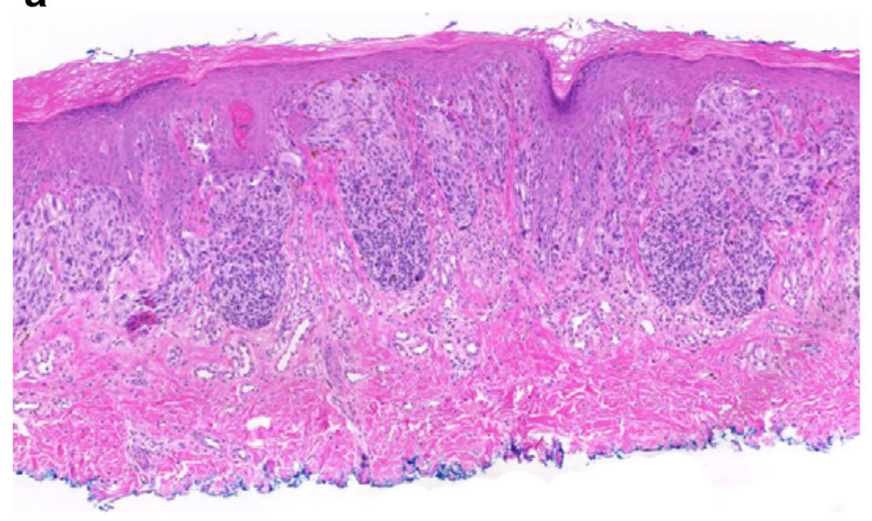

\section{C}

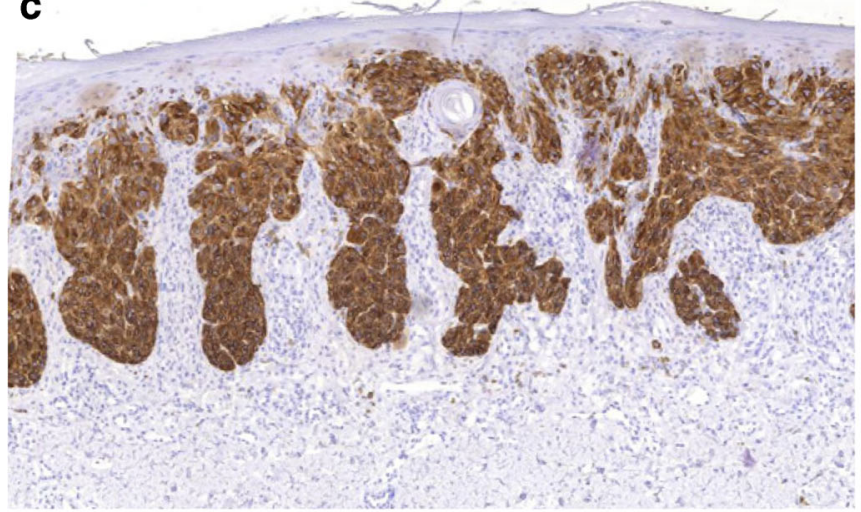

b

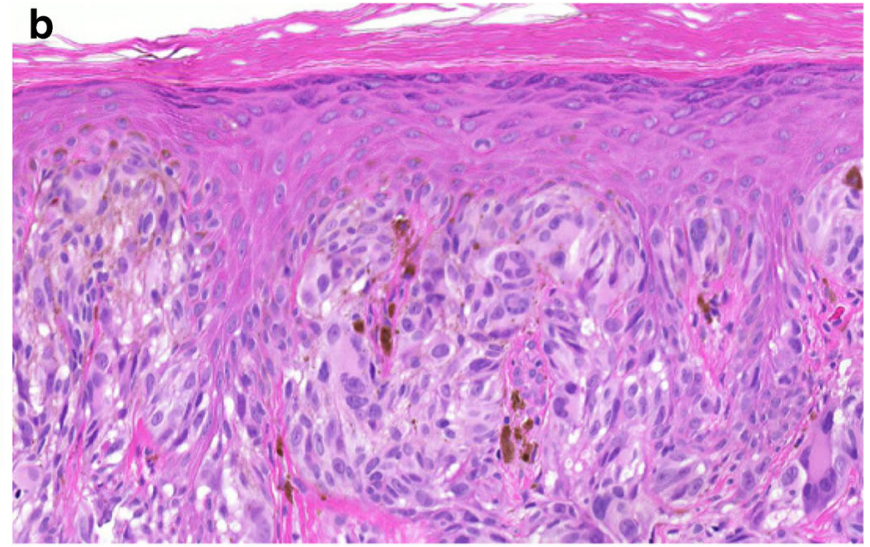

d

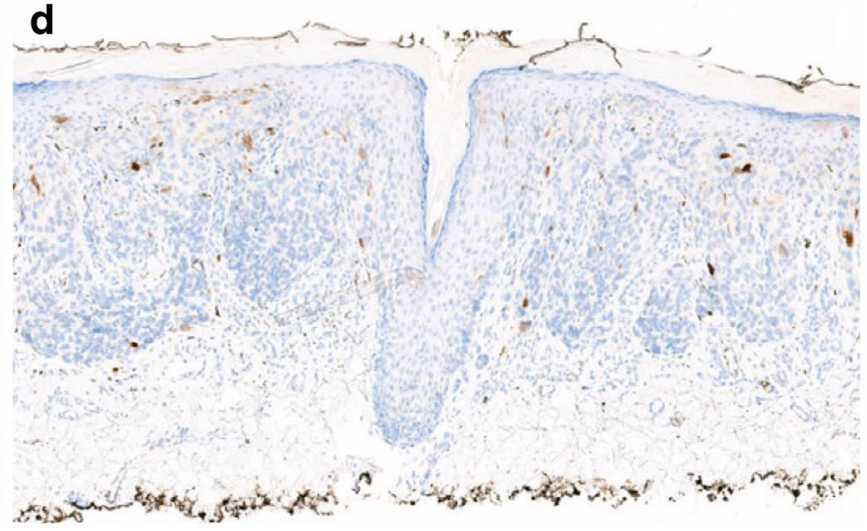

e

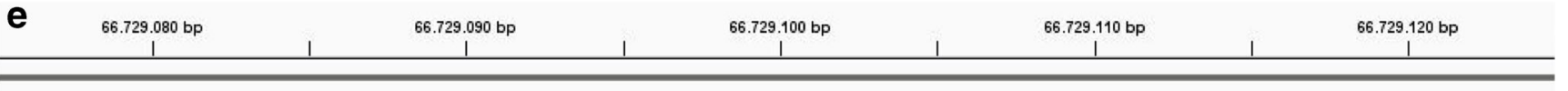

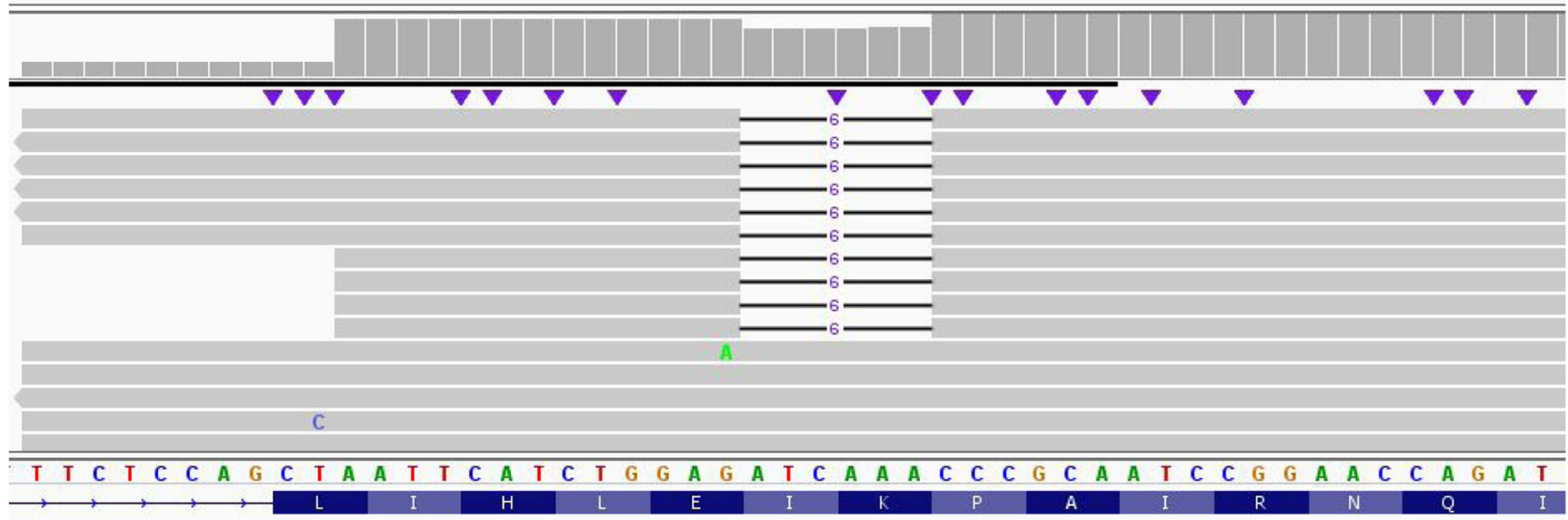

MAP2K1

Fig. 1 Microscopical and molecular findings case 2. a H\&E, $\times 5$ : relatively symmetrical, compound melanocytic lesion extending into the superficial dermis with large nests at the junction. b H\&E, $\times 40$ : epithelioid to spindle-shaped melanocytes with pale, eosinophilic cytoplasm. Variation in size and shape of the cells was encountered with large nuclei with prominent nucleoli and nuclear vacuoles. A few

The third case showed no signs of malignancy by histology, immunohistochemistry, and additional molecular testing. It was classified as a variant Spitz nevus. This lesion showed a different activating $M A P 2 K 1$ mutation, namely a missense multinucleated cells were seen, $\mathbf{c}$ Melan A stain, $\times 5$ : diffusely positive. d p16 stain, $\times 5$ : negative in the majority of the lesion. e Integrative Genomics Viewer (IGV) visualization of the sequence data containing MAP2K1 in-frame deletion c.307_312del (p.Ile103_Lys104del) in 24\% of the reads (RefSeq NM_002755.3). The black lines indicate the location of the deletion

mutation: c.169A $>\mathrm{G}$ (p.Lys57Glu). This mutation has been described to disrupt the negative regulatory region of MEK1, resulting in increased ERK1/2 activation [14]. 
a

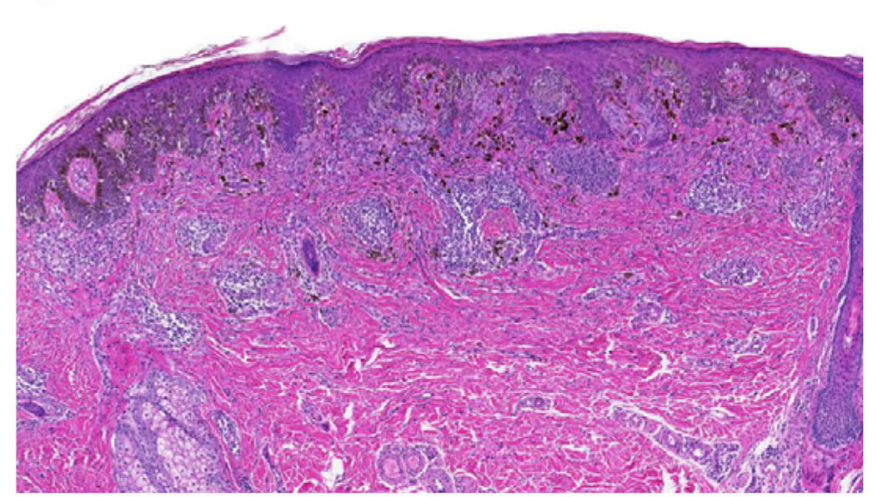

C

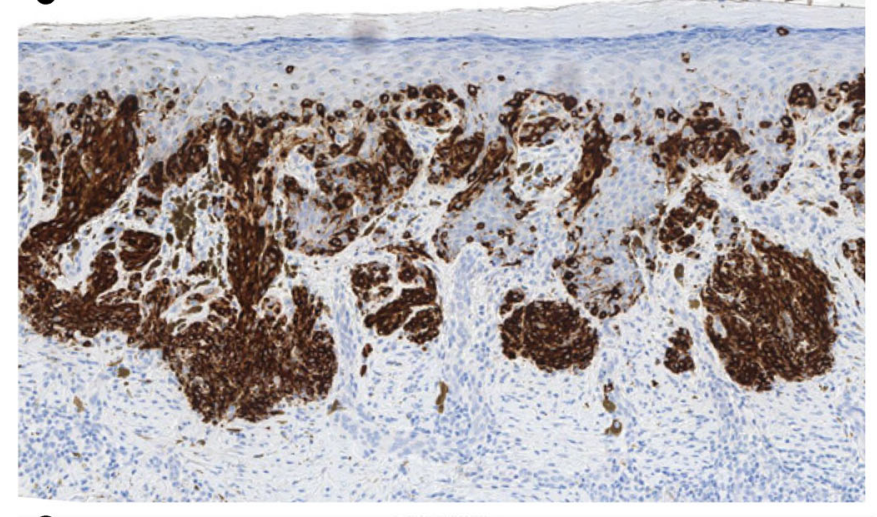

e

$66.727 .440 \mathrm{bp}$

$66.727 .460 \mathrm{bp}$

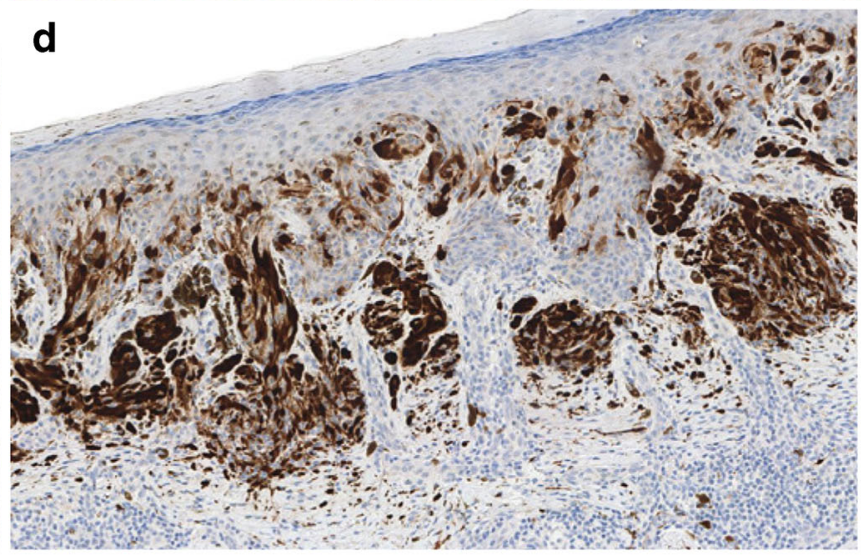

$66.727 .480 \mathrm{bp}$
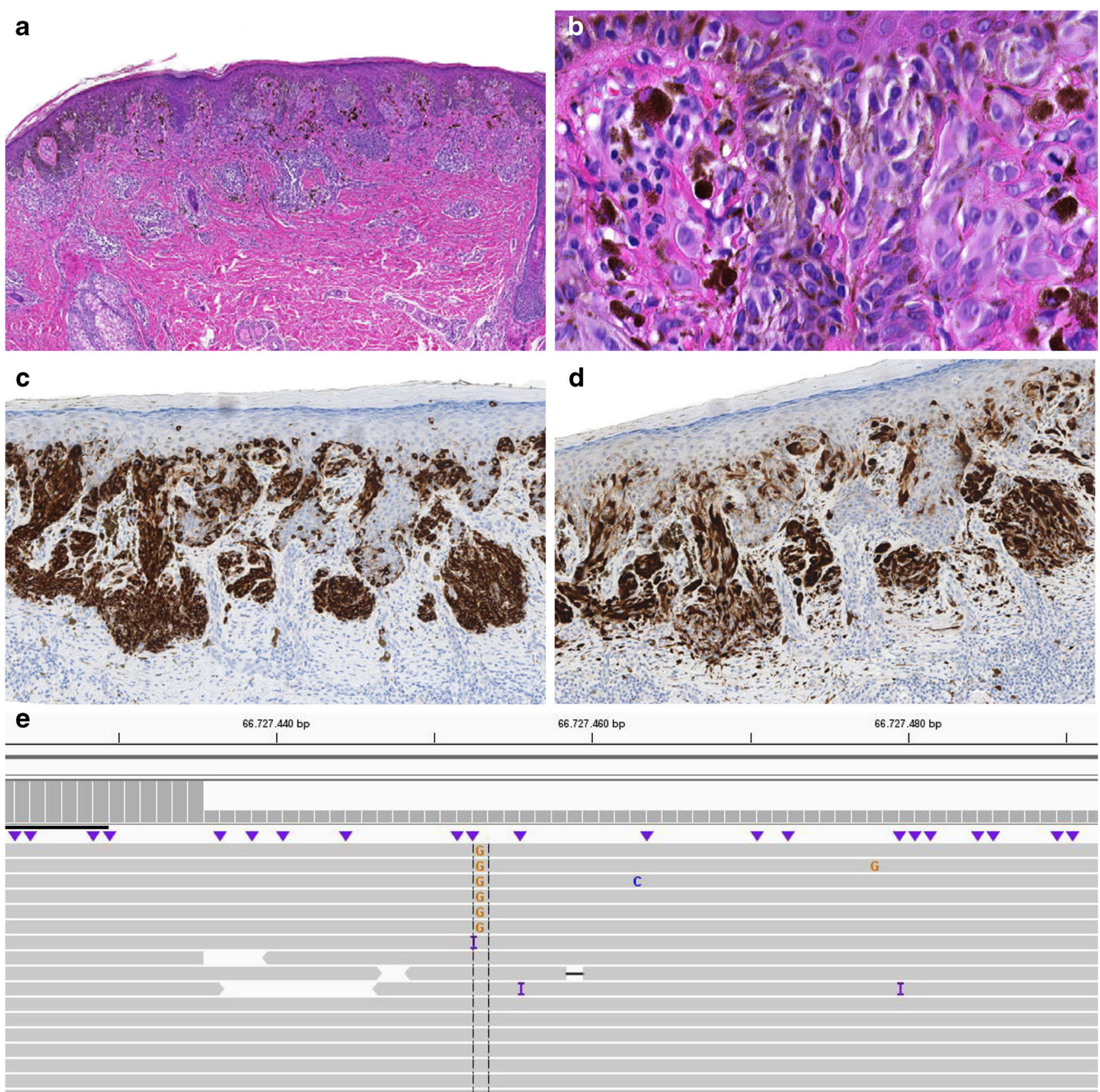

:GAAAGCGCCTTGAGGCCTITCTTACCCAGAAGCAGAAGGTGGGAGAACTGAAGGATGACGACTTIGAGA

MAP2K1

Fig. 2 Microscopical and molecular findings case 3. a H\&E, $\times 5$ : symmetrical, compound melanocytic lesion with nests and solitary melanocytes at the junction and a partly naevoid aspect of the dermal component. At the base melanocytes were spreading out between desmoplastic stroma. b H\&E, $\times 40$ : epithelioid to slightly spindleshaped melanocytes with pale, eosinophilic, partly pigmented cytoplasm.

Although this specific mutation has not been reported in spitzoid lesions, it has been described in melanomas [14, 15].

Based on these four cases, we hypothesize that in a part of the spitzoid neoplasms, a mutation in $M A P 2 K 1$ is the
The cells had enlarged nuclei and presence of nucleoli was encountered. c Melan A stain, $\times 5$ : diffusely positive showing focal ascension. d p16 stain, $\times 5$ : positive in a checkerboard pattern. e Integrative Genomics Viewer (IGV) visualization of the sequence data containing MAP2KI mutation c.169A $>\mathrm{G}$ (p.Lys57Glu) in 7\% of the reads (RefSeq NM 002755.3)

initiating genomic event. MAP2K1 mutations seem to be associated with intermediate or malignant spitzoid tumors both in our own cases and in a previously reported case [4]. This is also supported by the description of a case of 
spitzoid melanoma by Raghavan et al. [9]. They reported a $M A P 2 K 1$ mutation co-occurring with an HRAS mutation, mutations in CDKN2A, ARID1A, and NOTCH2, and a gain of chromosome $6 \mathrm{p}$. Also, they described one melanoma case with an activating $M A P 2 K 1$ mutation with additional mutations in PTEN, CDK4, ARID2, ATRX, and TP53 and a deletion of chromosome $6 \mathrm{p}$. This case was not classified as a spitzoid melanoma, but based on our findings, this lesion could possibly also have a spitzoid signature. However, not all spitzoid lesions with a MAP $2 K 1$ mutation show an intermediate or malignant phenotype since two of our cases with a mutation in this gene were classified as (probably) benign.

In conclusion, we describe four melanocytic lesions with spitzoid morphology harboring a MAP2K1 mutation. Our small series and the few recently reported cases show that $M A P 2 K 1$ mutations can indicate a spitzoid genetic signature of a melanocytic lesion. In addition, we show that MAP2K1 mutations can also be present in benign spitzoid lesions, but based on current limited data, MAP $2 K 1$ mutations seem more frequently present in AST and MST. These data can possibly assist in further unraveling the molecular background of spitzoid neoplasms assisting in more objectively classifying these lesions and, eventually, better patient management preventing overtreatment or undertreatment.

Acknowledgments We would like to thank D.P. Hayes (pathologist at the Meander Medical Center, Amersfoort, The Netherlands) for sending his case in consultation to the Pathology Department of the University Medical Center in Utrecht (UMCU).

Authors' contributions Design of the study: W.A.M. Blokx.

Writing - original draft preparation: K.G.P. Kerckhoffs, A.M.L. Jansen, W.A.M. Blokx.

Writing - review and editing: K.G.P. Kerckhoffs, T. Aallali, C.A. Ambarus, V. Sigurdsson, A.M.L. Jansen, W.A.M. Blokx.

Providing patient data: T. Aallali, C.A. Ambarus, V. Sigurdsson, W.A.M. Blokx.

Performance and interpretation of the molecular analysis: A.M.L. Jansen.

\section{Compliance with ethical standards}

Conflict of interest The authors declare that they have no conflict of interest.

Ethics approval Not applicable.

Consent to participate Not applicable.

Consent for publication The patients gave permission to use their clinical pictures.

Open Access This article is licensed under a Creative Commons Attribution 4.0 International License, which permits use, sharing, adaptation, distribution and reproduction in any medium or format, as long as you give appropriate credit to the original author(s) and the source, provide a link to the Creative Commons licence, and indicate if changes were made. The images or other third party material in this article are included in the article's Creative Commons licence, unless indicated otherwise in a credit line to the material. If material is not included in the article's Creative Commons licence and your intended use is not permitted by statutory regulation or exceeds the permitted use, you will need to obtain permission directly from the copyright holder. To view a copy of this licence, visit http://creativecommons.org/licenses/by/4.0/.

\section{References}

1. Bastian BC, LeBoit PE, Pinkel D (2000) Mutations and copy number increase of HRAS in Spitz nevi with distinctive histopathological features. Am J Pathol 157(3):967-972. https://doi.org/10.1016/ s0002-9440(10)64609-3

2. Quan VL, Panah E, Zhang B, Shi K, Mohan LS, Gerami P (2019) The role of gene fusions in melanocytic neoplasms. J Cutan Pathol 46(11):878-887. https://doi.org/10.1111/cup.13521

3. Shalin SC (2017) A review of kinase fusions in melanocytic tumors. Lab Investig 97(2):158-165. https://doi.org/10.1038/ labinvest.2016.122

4. Quan VL, Zhang B, Mohan LS, Shi K, Isales MC, Panah E, Taxter TJ, Beaubier N, White K, Gerami P (2019) Activating structural alterations in MAPK genes are distinct genetic drivers in a unique subgroup of spitzoid neoplasms. Am J Surg Pathol 43(4):538-548. https://doi.org/10.1097/pas.0000000000001213

5. Emery CM, Vijayendran KG, Zipser MC, Sawyer AM, Niu L, Kim JJ, Hatton C, Chopra R, Oberholzer PA, Karpova MB, MacConaill LE, Zhang J, Gray NS, Sellers WR, Dummer R, Garraway LA (2009) MEK1 mutations confer resistance to MEK and B-RAF inhibition. Proc Natl Acad Sci U S A 106(48):20411-20416. https://doi.org/10.1073/pnas.0905833106

6. Pritchard AL, Hayward NK (2013) Molecular pathways: mitogenactivated protein kinase pathway mutations and drug resistance. Clin Cancer Res 19(9):2301-2309. https://doi.org/10.1158/10780432.ccr-12-0383

7. Emelyanova M, Ghukasyan L, Abramov I, Ryabaya O, Stepanova E, Kudryavtseva A, Sadritdinova A, Dzhumakova C, Belysheva T, Surzhikov S, Lyubchenko L, Zasedatelev A, Nasedkina T (2017) Detection of BRAF, NRAS, KIT, GNAQ, GNA11 and MAP2K1/2 mutations in Russian melanoma patients using LNA PCR clamp and biochip analysis. Oncotarget 8(32):52304-52320. https://doi. org/10.18632/oncotarget. 17014

8. Kunz M, Dannemann M, Kelso J (2013) High-throughput sequencing of the melanoma genome. Exp Dermatol 22(1):10-17. https:// doi.org/10.1111/exd.12054

9. Raghavan SS, Peternel S, Mully TW, North JP, Pincus LB, LeBoit PE, McCalmont TH, Bastian BC, Yeh I (2020) Spitz melanoma is a distinct subset of spitzoid melanoma. Mod Pathol 33:1122-1134. https://doi.org/10.1038/s41379-019-0445-Z

10. Yeh I, Lang UE, Durieux E, Tee MK, Jorapur A, Shain AH, Haddad V, Pissaloux D, Chen X, Cerroni L, Judson RL, LeBoit PE, McCalmont TH, Bastian BC, de la Fouchardiere A (2017) Combined activation of MAP kinase pathway and beta-catenin signaling cause deep penetrating nevi. Nat Commun 8(1):644. https:// doi.org/10.1038/s41467-017-00758-3

11. Takata M, Suzuki T, Ansai S, Kimura T, Shirasaki F, Hatta N, Saida T (2005) Genome profiling of melanocytic tumors using multiplex ligation-dependent probe amplification (MLPA): its usefulness as an adjunctive diagnostic tool for melanocytic tumors. J Dermatol Sci 40(1):51-57. https://doi.org/10.1016/j.jdermsci.2005. 06.009 
12. Bastian BC, Xiong J, Frieden IJ, Williams ML, Chou P, Busam K, Pinkel D, LeBoit PE (2002) Genetic changes in neoplasms arising in congenital melanocytic nevi: differences between nodular proliferations and melanomas. Am J Pathol 161(4):1163-1169. https:// doi.org/10.1016/s0002-9440(10)64393-3

13. Elder DE (2018) WHO classification of skin tumours, 4th edn. IARC Press, Lyon

14. Carlino MS, Fung C, Shahheydari H, Todd JR, Boyd SC, Irvine M, Nagrial AM, Scolyer RA, Kefford RF, Long GV, Rizos H (2015) Preexisting MEK1P124 mutations diminish response to BRAF inhibitors in metastatic melanoma patients. Clin Cancer Res 21(1): 98-105. https://doi.org/10.1158/1078-0432.ccr-14-0759
15. Garman B, Anastopoulos IN, Krepler C, Brafford P, Sproesser K, Jiang Y, Wubbenhorst B, Amaravadi R, Bennett J, Beqiri M, Elder D, Flaherty KT, Frederick DT, Gangadhar TC, Guarino M, Hoon D, Karakousis G, Liu Q, Mitra N, Petrelli NJ, Schuchter L, Shannan B, Shields CL, Wargo J, Wenz B, Wilson MA, Xiao M, Xu W, Xu X, Yin X, Zhang NR, Davies MA, Herlyn M, Nathanson KL (2017) Genetic and genomic characterization of 462 melanoma patientderived xenografts, tumor biopsies, and cell lines. Cell Rep 21(7): 1936-1952. https://doi.org/10.1016/j.celrep.2017.10.052

Publisher's note Springer Nature remains neutral with regard to jurisdictional claims in published maps and institutional affiliations. 